\title{
Stereoselective Rh-Catalyzed Hydrogenation of Cyclobutyl Chiral Enamides: Double Stereodifferentiation vs Catalyst-Controlled Diastereoselection.
}

G. P. Aguado, A. G. Moglioni, E. García-Expósito, V. Branchadell, R. M. Ortuño*

\section{SUPPORTING INFORMATION}

General experimental methods $\mathrm{S} 2-\mathrm{S} 3$

Fully description of compounds 15b, 16a, 16b, 17a, 17b, 18a, 18b, 19a, 19b, 20a, 20b, 21a, 21b, 22a and 22b S4-S8

Computational details S9

Cartesian coordinates of optimized structures for model $(Z)$ and $(E)$-enamides $(\mathrm{R}=\mathrm{H})$, $(Z)-5,(Z)-7,(Z)-8,(E)-8$ S10-S15

Figure S1. Energy curve corresponding to rotation around $\mathrm{C}_{3}-\mathrm{C}_{4}$ bond obtained at the AM1 level of calculation for a model $(E)$-enamide with $\mathrm{R}=\mathrm{H}$ S16 


\section{General Experimental Methods}

The new enamides 7 and $(E)-8$ were prepared following the general procedures previously reported in the literature. ${ }^{11}$ Enamide $(E)-8$ was obtained as a minor product along with the major (Z)-isomer and isolated by column chromatography. Enamide 9 was prepared according to Scheme 4, following the protocols described below. General procedures for the hydrogenations with different catalysts are provided. All new products are described. Flash column chromatography was carried out on neutral Baker silica gel $(40 \mu \mathrm{m})$. Melting points were determined on a hot stage and are uncorrected. Distillation of small amounts of products was carried out in a bulb-to-bulb apparatus and oven temperature (ot) is given. Standard ${ }^{1} \mathrm{H}$ NMR and ${ }^{13} \mathrm{C}$ NMR spectra were recorded at 250 and $62.5 \mathrm{MHz}$, respectively, unless otherwise stated. Chemical shifts in NMR spectra are given in the $\delta$ scale. Electron impact HRMS spectra were recorded at $70 \mathrm{eV}$.

General Procedures for the Catalytic Hydrogenations. (a) With $\mathrm{RhCl}\left(P \mathrm{Ph} \mathrm{h}_{3}\right)_{3}$ (Wilkinson Catalyst). A mixture of the substrate and the catalyst (35 $\mathrm{mg}$ of catalyst per mmol of substrate) in 1:1 benzene-EtOH was stirred under hydrogen atmosphere at 4 atmospheres pressure for 11 days. Solvent was removed and the residue was purified by

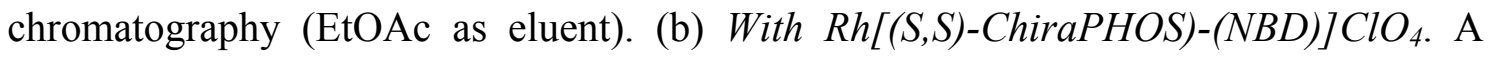
mixture of the substrate and the catalyst (15 $\mathrm{mg}$ of catalyst per mmol of substrate) in EtOH was stirred under hydrogen atmosphere at 4 atmospheres pressure for 3 days. Solvent was removed and the residue was chromatographed (EtOAc as eluent). (c) With $(C O D) R h(R, R)-\quad$ and $\quad[(C O D) R h(S, S)-E t-D u P H O S)] O T f, \quad$ and $\quad[(C O D) R h(R, R)-M e-$ DuPHOS)]OTf. A mixture of the substrate and the catalyst (24 $\mathrm{mg}$ of catalyst per mmol 
of substrate) in EtOH was stirred under hydrogen atmosphere at 2 atmospheres pressure for 1 day. Solvent was removed and the residue was chromatographed $\left(1: 1 \mathrm{CH}_{2} \mathrm{Cl}_{2-}\right.$ EtOAc as eluent).

Diastereomers resulting from the catalytic hydrogenation in each instance were isolated by column chromatography when possible. Otherwise, spectroscopic data for the diastereomeric mixture are described. 
(1'S,2S,3'R)-2-Acetylamino-4-(3'-acetyl-2',2'-dimethylcyclobutyl)butanoate, $15 \mathrm{~b}$.

Oil; $[\alpha]_{\mathrm{D}}-24\left(c\right.$ 0.25, MeOH); IR (film) 3302 (broad), 1745, 1700, $1660 \mathrm{~cm}^{-1} ; 500$ $\mathrm{MHz}{ }^{1} \mathrm{H}$ NMR (acetone- $\left.d_{6}\right) 0.80(\mathrm{~s}, 3 \mathrm{H}), 1.28$ (s, 3H), 1.31-2.10 (complex absorption, 7H), $1.90(\mathrm{~s}, 3 \mathrm{H}), 1.97(\mathrm{~s}, 3 \mathrm{H}), 2.85-2.88(\mathrm{~m}, 1 \mathrm{H}), 3.64(\mathrm{~s}, 3 \mathrm{H}), 4.35-4.41(\mathrm{~m}, 1 \mathrm{H}), 7.38$ (broad s); ${ }^{13} \mathrm{C}$ NMR (acetone- $d_{6}$ ) 16.2, 21.6, 22.8, 25.8, 29.2, 29.7, 29.6, 41.3, 42.5, 51.1, 52.0, 53.2, 169.0, 172.5, 204.8. HRMS (EI, $70 \mathrm{eV}$ ) calcd for $\mathrm{C}_{15} \mathrm{H}_{25} \mathrm{NO}_{4}(\mathrm{M})$ 283.1784, found 283.1788; calcd for $\mathrm{C}_{13} \mathrm{H}_{22} \mathrm{NO}_{3}$ [M- $\left.\mathrm{C}_{2} \mathrm{H}_{3} \mathrm{O}\right]$ 240.1599, found: 240.1593.

\section{Methyl}

$(1 ' R, 2 R, 3 ' S)$-2-acetylamino-4-(3'-acetyl-2',2'-

dimethylcyclobutyl)butanoate, 16a. Oil; $[\alpha]_{\mathrm{D}}+24.7(c 0.85, \mathrm{MeOH})$; IR (film) 3303 (broad), 1746, 1702, $1659 \mathrm{~cm}^{-1} ; 500 \mathrm{MHz}{ }^{1} \mathrm{H}$ NMR (acetone- $\left.d_{6}\right) 0.80(\mathrm{~s}, 3 \mathrm{H}), 1.28$ (s, 3H), 1.31-2.10 (complex absorption, 7H), $1.90(\mathrm{~s}, 3 \mathrm{H}), 1.97(\mathrm{~s}, 3 \mathrm{H}), 2.85-2.88(\mathrm{~m}, 1 \mathrm{H})$, $3.64(\mathrm{~s}, 3 \mathrm{H}), 4.35-4.41(\mathrm{~m}, 1 \mathrm{H}), 7.38$ (broad s); ${ }^{13} \mathrm{C}$ NMR (acetone- $\left.d_{6}\right) 16.2,21.6,22.8$, 25.8, 29.2, 29.7, 29.6, 41.3, 42.5, 51.1, 52.0, 53.2, 169.0, 172.5, 204.8; HRMS (EI, 70 eV) calcd for $\mathrm{C}_{15} \mathrm{H}_{25} \mathrm{NO}_{4}(\mathrm{M})$ 283.1784, found: 283.1779; calcd for $\mathrm{C}_{14} \mathrm{H}_{22} \mathrm{NO}_{4}$ [M$\left.\mathrm{CH}_{3}\right]$ 268.1549, found: 268.1563 .

\section{Methyl}

(1'R,2S,3'S)-2-acetylamino-4-(3'-acetyl-2',2'-

dimethylcyclobutyl)butanoate, 16b. Crystals; mp 89-91 ${ }^{\circ} \mathrm{C}$ (from $\mathrm{MeOH}$ ); $[\alpha]_{\mathrm{D}}+19.3$ (c $0.72, \mathrm{MeOH}$ ); IR (film) 3303 (broad), 1746, 1702, $1659 \mathrm{~cm}^{-1} ; 500 \mathrm{MHz}{ }^{1} \mathrm{H}$ NMR (acetone- $\left.d_{6}\right) 0.79(\mathrm{~s}, 3 \mathrm{H}), 1.27(\mathrm{~s}, 3 \mathrm{H}), 1.31-2.10$ (complex absorption, 7H), 1.90 (s, 3H), 1.97 (s, 3H), $2.87\left(\mathrm{dd}, J=7.9 \mathrm{~Hz}, J^{\prime}=9.8 \mathrm{~Hz}, 1 \mathrm{H}\right), 3.65(\mathrm{~s}, 3 \mathrm{H}), 4.35-4.40(\mathrm{~m}$, 1H), 7.32 (broad s, $1 \mathrm{H}) ;{ }^{13} \mathrm{C}$ NMR (acetone- $d_{6}$ ) 16.7, 22.1, 23.1, 26.2, 30.1, 30.6, 30.4, 41.8, 42.9, 51.6, 52.6, 53.7, 169.5, 173.0, 206.4. Anal. Calcd for $\mathrm{C}_{15} \mathrm{H}_{25} \mathrm{NO}_{4}$ : C, 63.58; H, 8.89; N, 4.94; Found: C, 63.37; H, 9.27; N, 4.39. 
Mixture of methyl $\left(1^{\prime} R, 2 R, 3^{\prime} R\right)-$ and $\left(1 ' R, 2 S, 3^{\prime} R\right)-2$-acetylamino-3-(2',2'dimethyl-3'-methoxycarbonylcyclobutyl)-propanoate, 17a and 17b. IR (film) 3307, 1746, 1660, $1554 \mathrm{~cm}^{-1} ;{ }^{1} \mathrm{H}$ NMR (acetone- $\left.d_{6}\right) 0.87$ (s, 17a), 0.89 (s, 17b), 1.15 (s, 17b), 1.16 (s, 17a), 1.60-2.11 (complex absorption, 17a+17b), 1.89 (s, 17a), 1.90 (s, 17b), 2.67-2.75 (m, 17a+17b), $3.60(\mathrm{~s}, \mathbf{1 7} \mathbf{a}+\mathbf{1 7 b}), 3.65(\mathrm{~s}, \mathbf{1 7 b}), 3.66(\mathrm{~s}, \mathbf{1 7 a}), 4.26-4.36(\mathrm{~m}$, 17a), 4.37-4.43 (m, 17b), 7.30 (broad s, 17a+17b); ${ }^{13} \mathrm{C}$ NMR (acetone- $\left.d_{6}\right) 16.7$ (17b), 16.9 (17a), 21.7 (17a+17b), 24.4 (17a), 24.7 (17b), 30.3 (17a+17b), 32.3 (17b), 32.5 (17a), 38.6 (17a), 38.8 (17b), 42.0 (17a), 42.3 (17b), 45.5 (17a), 45.9 (17b), 50.2 $(\mathbf{1 7} \mathbf{a}+\mathbf{1 7 b}), 50.4(\mathbf{1 7} \mathbf{a}+\mathbf{1 7 b}), 51.1$ (17b), $51.2(\mathbf{1 7 a}), 169.0(\mathbf{1 7} \mathbf{a}+\mathbf{1 7 b}), 172.6(\mathbf{1 7 a})$, 172.7 (17b). Anal. Calcd for $\mathrm{C}_{14} \mathrm{H}_{23} \mathrm{NO}_{5}$ : C, 58.93; H, 8.12; N, 4.93. Found: C, 58.71; $\mathrm{H}, 8.01 ; \mathrm{N}, 4.51$.

Mixture of methyl $\left(1^{\prime} R, 2 R, 3^{\prime} R\right)-$ and methyl $\left(1^{\prime} R, 2 S, 3\right.$ ' $\left.R\right)$-2benzyloxycarbonylamino-3-(2',2'-dimethyl-3'-methoxycarbonylcyclobutyl)propanoate, 18a and 18b IR (film) $3335,1720 \mathrm{~cm}^{-1}$; $500 \mathrm{MHz}{ }^{1} \mathrm{H}$ NMR (acetone- $d_{6}$ ) 0.88 (s, 18a), 0.91 (s, 18b), 1.15 (s, 18a), 1.16 (s, 18b), 1.61-2.17 (complex absorption, 18a+18b), $2.72\left(\mathrm{dd}, J=7.7 \mathrm{~Hz}, J^{\prime}=10.1 \mathrm{~Hz}, \mathbf{1 8 a}+\mathbf{1 8 b}\right), 3.59$ (s, 18b), 3.60 (s, 18a), $3.67(\mathrm{~s}, \mathbf{1 8 a}+\mathbf{1 8 b}), 4.09\left(\mathrm{dd}, J^{\prime}=6.3 \mathrm{~Hz}, J^{\prime}=8.4 \mathrm{~Hz}, \mathbf{1 8 a}\right), 4.16\left(\mathrm{dd}, J=4.5 \mathrm{~Hz}, J^{\prime}=9.4\right.$ $\mathrm{Hz}, \mathbf{1 8 b}), 5.06$ (s, 18a+18b), 7.28-7.41 (complex absorption, 18a+18b); ${ }^{13} \mathrm{C} \mathrm{NMR}$ (acetone- $\left.d_{6}\right) 16.7$ (18b), 17.0 (18a), 24.2 (18a), 24.7 (18b), 29.1 (18a), $29.6(\mathbf{1 8 b}), 32.3$ (18a+18b), 38.5 (18a), 38.7 (18b), 42.0 (18a), 42.0 (18b), 45.4 (18a), 45.9 (18b), 50.2 $(\mathbf{1 8 a} \mathbf{a} \mathbf{1 8 b}), 51.3(\mathbf{1 8 a}+\mathbf{1 8 b}), 52.4$ (18a), 53.1 (18b), 65.8 (18a+18b), 127.7, 128.2 $(\mathbf{1 8 a}+\mathbf{1 8 b}), 137.2(\mathbf{1 8 a}+\mathbf{1 8 b}), 156.0(\mathbf{1 8 a}+\mathbf{1 8 b}), 172.3(\mathbf{1 8 a}+\mathbf{1 8 b}), 172.7(\mathbf{1 8 a}+\mathbf{1 8 b})$. Anal. Calcd for $\mathrm{C}_{20} \mathrm{H}_{27} \mathrm{O}_{6} \mathrm{~N}$ : C, 63.64; H, 7.21; N, 3.71. Found: C, 63.83; H, 7.14; N, 3.59 . 
dimethylcyclobutyl)propanoate, 19a. Crystals; mp $120-125^{\circ} \mathrm{C}$ (acetone/pentane); $[\alpha]_{\mathrm{D}}$ -8.8 (c 0.68, MeOH); IR (film) 3302 (broad), 1746, $1660 \mathrm{~cm}-1 ;{ }^{1} \mathrm{H}$ NMR (acetone- $d_{6}$ ) $0.81(\mathrm{~s}, 3 \mathrm{H}), 1.27(\mathrm{~s}, 3 \mathrm{H}), 1.50-2.10$ (complex absorption, 5H), $1.89(\mathrm{~s}, 3 \mathrm{H}), 1.98(\mathrm{~s}$, 3H), 2.80-2.93 (dd, $\left.J=7.9 \mathrm{~Hz}, J^{\prime}=9.7 \mathrm{~Hz}, 1 \mathrm{H}\right), 3.65(\mathrm{~s}, 3 \mathrm{H}), 4.30-4.38(\mathrm{~m}, 1 \mathrm{H}), 7.30$ (broad s, $1 \mathrm{H}$ ); ${ }^{13} \mathrm{C}$ NMR (acetone- $d_{6}$ ) 17.4, 22.6, 23.8, 30.1, 30.2, 33.1, 39.1, 43.3, 51.3, 52.1, 53.9, 170.0, 173.6, 206.4. Anal. Calcd for $\mathrm{C}_{14} \mathrm{H}_{23} \mathrm{NO}_{4}$ : C, 62.40; H, 8.61; N, 5.20. Found: C, 62.41; H, 8.61; N, 5.24.

\section{Methyl (1'R,2S,3'R)-2-acetylamino-3-(3'-acetyl-2',2'-dimethylcyclobutyl)-}

propanoate, 19b. Spectra recorded from a enriched fraction. IR (film) 3302, 1746, 1700, $1660 \mathrm{~cm}^{-1}$; ${ }^{1} \mathrm{H}$ NMR (acetone- $\left.d_{6}\right) 0.83(\mathrm{~s}, 3 \mathrm{H}), 1.27$ (s, 3H), 1.50-2.10 (complex absorption, 5H), 1.90 (s, 3H), $1.98(\mathrm{~s}, 3 \mathrm{H}), 2.80-2.93\left(\mathrm{dd}, J=7.9 \mathrm{~Hz}, J^{\prime}=9.7 \mathrm{~Hz}, 1 \mathrm{H}\right)$, $3.64(\mathrm{~s}, 3 \mathrm{H}), 4.38(\mathrm{~m}, 1 \mathrm{H}), 7.30($ broad s, $1 \mathrm{H}) ;{ }^{13} \mathrm{C}$ NMR (acetone- $\left.d_{6}\right) 17.0,22.6,24.1$, $30.1,30.2,32.9,39.3,43.5,51.3,51.8,52.1,170.0,173.7,206.4$.

Mixture of methyl $\left(1^{\prime} R, 2 R, 3^{\prime} R\right)$ - and methyl $\left(1^{\prime} R, 2 S, 3 ' R\right)-3-\left(3^{\prime}\right.$-acetyl-2',2'dimethylcyclobutyl)-2-benzyloxycarbonylamino-propanoate, 20a and 20b. IR (film) $3325,1702 \mathrm{~cm}^{-1} ; 500 \mathrm{MHz}{ }^{1} \mathrm{H}$ NMR (acetone- $d_{6}$ ) 0.82 (s, 20a), 0.84 (s, 20b), 1.26 (s, 20a), 1.27 (s, 20b), 1.55-2.13 (complex absorption, 20a+20b), 1.97 (s, 20b), 1.98 (s, 20a), $2.87\left(\mathrm{dd}, J=7.5 \mathrm{~Hz}, J^{\prime}=10.3 \mathrm{~Hz}, \mathbf{2 0 a}+\mathbf{2 0 b}\right), 3.66$ (s, 20b), 3.67 (s, 20a), $4.10\left(\mathrm{dd}, J=5.6 \mathrm{~Hz}, J^{\prime}=9.2 \mathrm{~Hz}, \mathbf{2 0 a}\right), 4.17\left(\mathrm{dd}, J=4.5 \mathrm{~Hz}, J^{\prime}=9.4 \mathrm{~Hz}, \mathbf{2 0 b}\right), 5.06-$ 5.08 (complex absorption, 20a+20b), $6.72(\mathrm{~d}, J=8.5 \mathrm{~Hz}, \mathbf{2 0 a}+\mathbf{2 0 b})$, 7.29-7.37 (complex absorption, 20a+20b); ${ }^{13} \mathrm{C}$ NMR (acetone- $\left.d_{6}\right)$ : 17.1 (20b), 17.4 (20a), 23.6 (20a), 24.0 (20b), 29.6, 29.7 (20a+20b), 32.7 (20a+20b), 39.0 (20a), 39.4 (20b), 43.6 (20a), 43.6 (20b), 52.2 (20b), 52.9 (20a), 53.9 (20a+20b), 54.5 (20b), 54.9 (20a), 66.6 (20b), 67.0 (20a), 128.5, 128.5, 129.1 (20a+20b), 137.6 (20a), 138.1 (20b), 156.8 
$(\mathbf{2 0 a}+\mathbf{2 0 b}), 173.4(\mathbf{2 0 a}+\mathbf{2 0 b}), 206.7(\mathbf{2 0 a}+\mathbf{2 0 b}) ;$ HRMS (EI, $70 \mathrm{eV})$ calcd for $\mathrm{C}_{20} \mathrm{H}_{27} \mathrm{NO}_{5}$ (M) 361.1889, found: 361.1891.

Mixture of methyl $\left(1^{\prime} R, 2 R, 3^{\prime} R\right)$ - and (1' $\left.R, 2 S, 3^{\prime} R\right)$-2-benzyloxycarbonylamino-3(2',2'-dimethyl-3'-methoxycarbonylcyclobutyl)propanoate, 21a and $21 \mathrm{~b}$. IR (film) 3341 (broad), $1717 \mathrm{~cm}^{-1} ; 500 \mathrm{MHz}{ }^{1} \mathrm{H}$ NMR (acetone- $d_{6}$ ) 0.88 (s, 21b), 0.91 (s, 21a), $1.15(\mathrm{~s}, \mathbf{2 1 b}), 1.16$ (s, 21a), 1.61-2.17 (complex absorption, 21a+21b), $2.72(\mathrm{dd}, J=7.7$ $\left.\mathrm{Hz}, J^{\prime}=10.1 \mathrm{~Hz}, 21 \mathbf{a}+21 \mathbf{b}\right), 3.59$ (s, 21a), 3.60 (s, 21b), 3.67 (s, 21a+21b), $4.09(\mathrm{dd}, J$ $\left.=6.3 \mathrm{~Hz}, J^{\prime}=8.4 \mathrm{~Hz}, \mathbf{2 1 b}\right), 4.16\left(\mathrm{dd}, J=4.5 \mathrm{~Hz}, J^{\prime}=9.4 \mathrm{~Hz}, \mathbf{2 1 a}\right), 5.06(\mathrm{~s}, \mathbf{2 1} \mathbf{a}+\mathbf{2 1} \mathbf{b})$, 7.28-7.41 (complex absorption, 21a+21b); ${ }^{13} \mathrm{C}$ RMN (acetone- $d_{6}$ ) 16.7 (21a), 17.0 (21b), 24.2 (21b), 24.7 (21a), 29.1 (21b), 29.6 (21a), 32.3 (21a+21b), 38.6 (21b), 38.7 (21a), 42.0 (21b), 42.0 (21a), 45.4 (21b), 45.9 (21a), 50.2 (21a+21b), 51.3 (21a+21b), 52.4 (21b), 53.1 (21a), $65.9(21 \mathbf{a}+21 \mathbf{b}), 127.7,128.2(\mathbf{2 1 a}+\mathbf{2 1 b}), 137.2(\mathbf{2 1} \mathbf{a}+\mathbf{2 1 b})$, $156.0(21 \mathbf{a}+21 \mathbf{b}), 172.3(\mathbf{2 1 a}+21 \mathbf{b}), 172.7(21 \mathbf{a}+21 \mathbf{b})$. Anal. Calcd for $\mathrm{C}_{20} \mathrm{H}_{27} \mathrm{O}_{6} \mathrm{~N}: \mathrm{C}$, 63.64; H, 7.21; N: 3.71. Found: C, 63.87; H, 7.46; N, 3.58.

Mixture of methyl (1'S,2R,3'S)- and (1'S,2S,3'S)-2-acetylamino-3-(2',2'-dimethyl3'-benzyloximethylcyclobutyl)-propanoate, 22a and 22b. IR (film) 3307, 1746, 1660 $\mathrm{cm}^{-1} ;{ }^{1} \mathrm{H}$ NMR (acetone- $\left.d_{6}\right) 0.96$ (s, 22b), 0.98 (s, 22a), 1.09 (s, 22a+22b), 1.15-1.42 $(\mathrm{m}, \mathbf{2 2} \mathbf{a}+\mathbf{2 2 b}), \quad 1.52-1.84(\mathrm{~m}, \mathbf{2 2 a}+\mathbf{2 2 b}), 1.92(\mathrm{~s}, \mathbf{2 2} \mathbf{a}+\mathbf{2 2 b}), 1.90-2.03$ (complex absorption, 22a $+\mathbf{2 2 b}$ ), 2.04-2.30 (complex absorption, 22a+22b), 3.31-3.52 (complex absorption, 22a+22b), 3.66 (s, 22a), 3.67 (s, 22b), 4.21-4.31 (m, 22b), 4.32-4.42 (m, 22a), 4.47 (s, 22a+22b), 7.23-7.39 (complex absorption, 22a+22b), 7.60 (broad s, 22a+22b); ${ }^{13} \mathrm{C}$ NMR (acetone- $\left.d_{6}\right) 16.2$ (22a), 16.3 (22b), $22.1(\mathbf{2 2 a} \mathbf{a}+\mathbf{2 2 b}), 26.6$ (22b), 26.9 (22a), 30.3 (22a+22b), 32.8 (22a $\mathbf{a}+\mathbf{2 2 b}), 39.2(\mathbf{2 2 a}+\mathbf{2 2 b}), 39.7$ (22b), 39.9 (22a), 42.1 (22b), 42.5 (22a), 50.9 (22a+22b), 51.4 (22a), 51.6 (22b), $71.2(\mathbf{2 2 a} \mathbf{a}+\mathbf{2 2} \mathbf{b}), 72.7$ $(\mathbf{2 2} \mathbf{a}+\mathbf{2 2 b}), 127.5,127.7,128.5(\mathbf{2 2} \mathbf{a}+\mathbf{2 2} \mathbf{b}), 139.6(\mathbf{2 2} \mathbf{a}+\mathbf{2 2} \mathbf{b}), 169.3(\mathbf{2 2} \mathbf{a}+\mathbf{2 2} \mathbf{b}), 173.4$ 
$(22 a+22 b)$. Anal. Calcd. for $\mathrm{C}_{20} \mathrm{H}_{29} \mathrm{O}_{4} \mathrm{~N}: \mathrm{C}, 69.14 ; \mathrm{H}, 8.41 ; \mathrm{N}, 4.03$. Found: $\mathrm{C}, 68.90 ; \mathrm{H}$, 8.63; N, 4.24. 
Computational Details. All calculations have been done using the Gaussian-98 program. ${ }^{1}$ A preliminary exploration of the potential energy surface has been done using the semiempirical AM1 method. ${ }^{2}$ Then, the structures of the most stable conformers have been fully optimized using the BPW91 density functional method ${ }^{3,4}$ with the 6 $31 \mathrm{G}(\mathrm{d})$ basis set. ${ }^{5}$ All structures have been characterized as energy minima through the calculation of the harmonic vibrational frequencies.

1. Gaussian 98, Revision A.9, Frisch, M. J.; Trucks, G. W.; Schlegel, H. B.; Scuseria, G. E.; Robb, M. A.; Cheeseman, J. R.; Zakrzewski, V. G.; Montgomery, Jr., J. A.; Stratmann, R. E.; Burant, J. C.; Dapprich, S.; Millam, J. M.; Daniels, A. D.; Kudin, K. N.; Strain, M. C.; Farkas, O.; Tomasi, J.; Barone, V.; Cossi, M.; Cammi, R.; Mennucci, B.; Pomelli, C.; Adamo, C.; Clifford, S.; Ochterski, J.; Petersson, G. A.; Ayala, P. Y.; Cui, Q.; Morokuma, K.; Malick, D. K.; Rabuck, A. D.; Raghavachari, K.; Foresman, J. B.; Cioslowski, J.; Ortiz, J. V.; Stefanov, B. B.; Liu, G.; Liashenko, A.; Piskorz, P.; Komaromi, I.; Gomperts, R.; Martin, R. L.; Fox, D. J.; Keith, T.; Al-Laham, M. A.; Peng, C. Y.; Nanayakkara, A.; Gonzalez, C.; Challacombe, M.; Gill, P. M. W.; Johnson, B.; Chen, W.; Wong, M. W.; Andres, J. L.; Gonzalez, C.; Head-Gordon, M.; Replogle, E. S.; Pople, J. A. Gaussian, Inc., Pittsburgh PA, 1998. http://www.gaussian.com.

2. Dewar, M. J. S.; Zoebisch, E. G.; Healy, E. F.; Stewart, J. J. P. J. Am. Chem. Soc. 1985, 107, 3902 .

3. Becke, A. D. Phys. Rev. A 1988, 38, 3098. 
4. (a) Wang, Y.; Perdew, J. P. Phys. Rev. B 1991, 44, 13298. (b) Perdew, J. P.; Chevary, J. A.; Vosko, S. H.; Jackson, K. A.; Pederson, M. R.; Singh, D. J.; Fiolhais, C. Phys. Rev. B 1992, 46, 6671.

5. (a) Ditchfield, R.; Hehre, W. J.; Pople, J. A. J. Chem. Phys. 1971, 54, 724. (b) Hehre, W. J.; Ditchfield, R.; Pople, J. A. J. Chem. Phys. 1972, 56, 2257. (c) Hariharan, P.C.; Pople, J. A. Theor. Chim. Acta 1973, 28, 213. 
Cartesian coordinates in $\AA$. Geometries and energies obtained at the BPW91/6-31G(d) level of calculation

(Z)-enamide with $\mathrm{R}=\mathrm{H}$.

$\mathrm{E}=-749.03576$ a.u.

$\begin{array}{lrrr}\mathrm{C} & 1.110668 & 0.021273 & -0.130801 \\ \mathrm{C} & -0.094859 & -0.567843 & -0.363521 \\ \mathrm{C} & -1.415568 & 0.041587 & -0.667329 \\ \mathrm{C} & -2.732933 & -0.491791 & 0.054534 \\ \mathrm{C} & -3.460919 & -0.358820 & -1.326211 \\ \mathrm{C} & -2.082053 & -0.349172 & -2.033199 \\ \mathrm{C} & -3.250722 & 0.385008 & 1.197017 \\ \mathrm{C} & -2.637245 & -1.959293 & 0.499328 \\ \mathrm{H} & -0.059280 & -1.660740 & -0.390748 \\ \mathrm{H} & -1.382889 & 1.133032 & -0.561945 \\ \mathrm{H} & -3.976978 & 0.610963 & -1.419981 \\ \mathrm{H} & -4.168801 & -1.159067 & -1.598596 \\ \mathrm{H} & -1.928487 & 0.353639 & -2.867085 \\ \mathrm{H} & -1.777282 & -1.354505 & -2.367031 \\ \mathrm{H} & -4.242009 & 0.035921 & 1.538176 \\ \mathrm{H} & -2.562561 & 0.362712 & 2.057539 \\ \mathrm{H} & -3.346094 & 1.436658 & 0.883337 \\ \mathrm{H} & -3.630094 & -2.321442 & 0.817718 \\ \mathrm{H} & -2.291024 & -2.624897 & -0.309843 \\ \mathrm{H} & -1.947572 & -2.076568 & 1.352505 \\ \mathrm{~N} & 1.414670 & 1.399750 & -0.020284 \\ \mathrm{C} & 2.373784 & -0.779836 & -0.063704 \\ \mathrm{O} & 3.490312 & -0.259796 & -0.016306 \\ \mathrm{O} & 2.179460 & -2.123585 & -0.089098 \\ \mathrm{C} & 3.392964 & -2.907428 & -0.062303 \\ \mathrm{H} & 3.060214 & -3.952608 & -0.079493 \\ \mathrm{H} & 3.969372 & -2.698007 & 0.851037 \\ \mathrm{H} & 4.018158 & -2.684040 & -0.939690 \\ \mathrm{H} & 2.418236 & 1.559404 & -0.146559 \\ \mathrm{C} & 0.730559 & 2.372446 & 0.700078 \\ \mathrm{O} & -0.393606 & 2.220696 & 1.178574 \\ \mathrm{C} & 1.510298 & 3.677477 & 0.858554 \\ \mathrm{H} & 0.817096 & 4.519483 & 0.720792 \\ \mathrm{H} & 1.903499 & 3.739695 & 1.886867 \\ \mathrm{H} & 2.350628 & 3.781958 & 0.154980\end{array}$

$\begin{array}{lrrr}\mathrm{C} & -2.906974 & -1.947650 & -0.372672 \\ \mathrm{C} & -2.092464 & -1.470402 & -1.602084 \\ \mathrm{C} & -3.670707 & 0.437545 & 0.207698 \\ \mathrm{C} & -2.128603 & -0.720102 & 1.829392 \\ \mathrm{H} & 0.136295 & -1.775791 & -0.055729 \\ \mathrm{H} & -1.436059 & 0.657594 & -1.144854 \\ \mathrm{H} & -3.976766 & -2.165645 & -0.527866 \\ \mathrm{H} & -2.447324 & -2.821616 & 0.118800 \\ \mathrm{H} & -2.722591 & -1.028001 & -2.389243 \\ \mathrm{H} & -1.417305 & -2.201359 & -2.075172 \\ \mathrm{H} & -4.552836 & 0.160178 & 0.811731 \\ \mathrm{H} & -3.315456 & 1.425223 & 0.541026 \\ \mathrm{H} & -4.001313 & 0.529971 & -0.841244 \\ \mathrm{H} & -2.994934 & -0.967267 & 2.468476 \\ \mathrm{H} & -1.369763 & -1.506144 & 1.978809 \\ \mathrm{H} & -1.701712 & 0.234421 & 2.174711 \\ \mathrm{~N} & 1.158433 & 1.447624 & -0.524339 \\ \mathrm{C} & 2.444185 & -0.510474 & -0.044539 \\ \mathrm{O} & 3.472030 & 0.158112 & -0.167684 \\ \mathrm{O} & 2.446737 & -1.831353 & 0.275389 \\ \mathrm{C} & 3.761111 & -2.402440 & 0.458552 \\ \mathrm{H} & 3.584655 & -3.456053 & 0.708116 \\ \mathrm{H} & 4.294221 & -1.893972 & 1.275698 \\ \mathrm{H} & 4.353767 & -2.315471 & -0.464360 \\ \mathrm{H} & 2.113287 & 1.727105 & -0.764597 \\ \mathrm{C} & 0.359551 & 2.438513 & 0.038248 \\ \mathrm{O} & -0.683020 & 2.219543 & 0.655245 \\ \mathrm{C} & 0.895478 & 3.853496 & -0.173370 \\ \mathrm{H} & 1.652967 & 3.926203 & -0.968934 \\ \mathrm{H} & 0.052824 & 4.516878 & -0.414746 \\ \mathrm{H} & 1.339734 & 4.214772 & 0.768974\end{array}$

(Z)-enamide with $\mathrm{R}=\mathrm{H}$. Secondary minimum

$\mathrm{E}=-749.03518$ a.u.
C 1.080473
0.055309
$-0.272707$
C -0.032546
$-0.725781$
$-0.314576$
C -1.413684
$-0.356437$
$-0.724687$
C -2.560270
$-0.612378$
0.365273 
(Z)-5

$\mathrm{E}=-976.89186$ a.u.

$\begin{array}{lrrr}\mathrm{C} & 2.207814 & -0.195594 & -0.194085 \\ \mathrm{C} & 0.877050 & -0.462552 & -0.085891 \\ \mathrm{C} & -0.300089 & 0.396876 & -0.379446 \\ \mathrm{C} & -1.513478 & 0.458484 & 0.649226 \\ \mathrm{C} & -2.477355 & 0.425342 & -0.617653 \\ \mathrm{C} & -1.306381 & -0.137347 & -1.452775 \\ \mathrm{C} & -1.592504 & 1.728413 & 1.497682 \\ \mathrm{C} & -1.636867 & -0.793512 & 1.529539 \\ \mathrm{H} & 0.649731 & -1.493861 & 0.197776 \\ \mathrm{H} & 0.006747 & 1.423377 & -0.614129 \\ \mathrm{H} & -2.736199 & 1.457715 & -0.898905 \\ \mathrm{C} & -3.736157 & -0.404923 & -0.536076 \\ \mathrm{H} & -1.183169 & 0.243086 & -2.478508 \\ \mathrm{H} & -1.348869 & -1.237057 & -1.481797 \\ \mathrm{H} & -2.555759 & 1.776304 & 2.035082 \\ \mathrm{H} & -0.778133 & 1.752175 & 2.239534 \\ \mathrm{H} & -1.495881 & 2.633994 & 0.878414 \\ \mathrm{H} & -2.572780 & -0.760103 & 2.112723 \\ \mathrm{H} & -1.649582 & -1.727661 & 0.946337 \\ \mathrm{H} & -0.799534 & -0.841100 & 2.245655 \\ \mathrm{~N} & 2.847675 & 1.022157 & -0.525839 \\ \mathrm{C} & 3.234100 & -1.280146 & -0.063716 \\ \mathrm{O} & 4.427855 & -1.098019 & -0.310389 \\ \mathrm{O} & 2.726802 & -2.478234 & 0.320451 \\ \mathrm{C} & 3.699452 & -3.543507 & 0.411377 \\ \mathrm{H} & 3.131076 & -4.423976 & 0.735194 \\ \mathrm{H} & 4.480360 & -3.292898 & 1.144543 \\ \mathrm{H} & 4.169540 & -3.722248 & -0.567117 \\ \mathrm{H} & 3.817733 & 0.853638 & -0.807729 \\ \mathrm{C} & 2.558778 & 2.301527 & -0.066733 \\ \mathrm{O} & 1.536703 & 2.604468 & 0.549870 \\ \mathrm{C} & 3.641086 & 3.329884 & -0.392422 \\ \mathrm{H} & 3.155698 & 4.247540 & -0.754932 \\ \mathrm{H} & 4.179920 & 3.588495 & 0.533947 \\ \mathrm{H} & 4.372304 & 2.989003 & -1.141429 \\ \mathrm{O} & -3.817490 & -1.607415 & -0.753015 \\ \mathrm{O} & -4.800475 & 0.359786 & -0.141717 \\ \mathrm{C} & -6.040045 & -0.366593 & 0.012989 \\ \mathrm{H} & -5.934866 & -1.162270 & 0.765962 \\ \mathrm{H} & -6.345135 & -0.821124 & -0.941763 \\ \mathrm{H} & -6.776511 & 0.379081 & 0.338320\end{array}$

$\begin{array}{lll}\text { C } & 0.056418 & -2.610854\end{array}$

C $\quad 1.232438 \quad-1.642271$

C $\quad-1.797388-1.443443$

C $\quad-1.664144 \quad-0.895979$

$\mathrm{H} \quad 0.311617 \quad 0.977653$

H $\quad 0.697296 \quad-0.536914$

H $\quad 0.171980 \quad-3.168336$

C $\quad-0.336292 \quad-3.559734$

$\mathrm{H} \quad 2.218093 \quad-1.921480$

H $\quad 1.330201 \quad-1.440162$

$\mathrm{H} \quad-2.627127 \quad-2.148702$

$\mathrm{H} \quad-2.233008 \quad-0.459543$

H $\quad-1.248933-1.779699$

H $-2.503339-1.584981$

H $\quad-1.045258 \quad-0.871212$

H $\quad-2.090075 \quad 0.108798$

$\begin{array}{lll}\mathrm{N} & 1.488829 & 2.205399\end{array}$

C $1.308835 \quad 3.239006$

O $\quad 1.885758 \quad 4.225084$

O 0.868109

C 1.136483

H 0.717298

$\mathrm{H} \quad 0.650770$

$\mathrm{H} \quad 2.219344$

H 2.056569

C 0.885326

O $\quad-0.025758$

C 1.454216

H 1.594682

H $\quad 0.718170$

H 2.408554

O -0.014381

C -1.170482

$\mathrm{H} \quad-2.035406$

$\mathrm{H} \quad-0.565382$

$\mathrm{H} \quad-1.515305$
0.981998

1.233328

$-0.511072$

1.948691

2.074518

$-0.597207$

0.036606

2.102966

0.829960

2.311264

$-0.324720$

$-0.748719$

$-1.405164$

2.144703

2.859229

1.789086

$-0.890835$

1.245362

0.782883

$3.165647 \quad 2.526157$

$4.340421 \quad 3.324385$

$4.117211 \quad 4.313107$

$5.224896 \quad 2.886252$

$4.522144 \quad 3.392840$

$3.055187-0.957950$

$1.780442-2.067871$

$\begin{array}{ll}0.953361 & -2.123408\end{array}$

$2.441588 \quad-3.322597$

$1.669840-4.093156$

$3.163761-3.712618$

$2.965351 \quad-3.159067$

$-3.348306 \quad 3.271159$

$-4.775043 \quad 1.714590$

$\begin{array}{ll}-4.479280 & 1.096041\end{array}$

$-5.463915 \quad 1.098342$

$-5.303490 \quad 2.613940$

(Z)-7

$\mathrm{E}=-901.66281$ a.u.

$\begin{array}{rrrr}\mathrm{C} & 1.060490 & 1.998232 & 0.442025 \\ \mathrm{C} & 0.581959 & 0.862797 & 1.021311 \\ \mathrm{C} & 0.460125 & -0.514249 & 0.473355 \\ \mathrm{C} & -0.877828 & -1.341581 & 0.707458\end{array}$


(Z)-8

$\mathrm{E}=-1055.46712$ a.u.

\begin{tabular}{rrrr}
$\mathrm{C}$ & 0.275711 & 2.655426 & -0.098358 \\
$\mathrm{C}$ & -0.007248 & 1.323257 & -0.119568 \\
$\mathrm{C}$ & 0.118271 & 0.337936 & -1.224686 \\
$\mathrm{C}$ & -1.040009 & -0.723511 & -1.494858 \\
$\mathrm{C}$ & 0.118759 & -1.762848 & -1.780624 \\
$\mathrm{C}$ & 1.093460 & -0.864349 & -0.985916 \\
$\mathrm{C}$ & -1.952515 & -0.382141 & -2.677643 \\
$\mathrm{C}$ & -1.881271 & -1.024338 & -0.244476 \\
$\mathrm{H}$ & -0.302536 & 0.915085 & 0.851013 \\
$\mathrm{H}$ & 0.355972 & 0.836060 & -2.172607 \\
$\mathrm{H}$ & 0.364822 & -1.703888 & -2.855240 \\
$\mathrm{C}$ & 0.045303 & -3.262100 & -1.455261 \\
$\mathrm{H}$ & 2.121613 & -0.750626 & -1.362244 \\
$\mathrm{H}$ & 1.125900 & -1.156296 & 0.075317 \\
$\mathrm{H}$ & -2.650970 & -1.210236 & -2.893341 \\
$\mathrm{H}$ & -2.548660 & 0.519119 & -2.461380 \\
$\mathrm{H}$ & -1.370651 & -0.175446 & -3.589951 \\
$\mathrm{H}$ & -2.558487 & -1.875336 & -0.423325 \\
$\mathrm{H}$ & -1.270400 & -1.282333 & 0.633072 \\
$\mathrm{H}$ & -2.505707 & -0.150055 & 0.007828 \\
$\mathrm{~N}$ & 0.674942 & 3.495911 & -1.165833 \\
$\mathrm{C}$ & 0.307510 & 3.426849 & 1.184509 \\
$\mathrm{O}$ & 0.713120 & 4.588989 & 1.252914 \\
$\mathrm{O}$ & -0.121811 & 2.724472 & 2.264313 \\
$\mathrm{C}$ & -0.058746 & 3.445297 & 3.514740 \\
$\mathrm{H}$ & -0.442596 & 2.746735 & 4.268463 \\
$\mathrm{H}$ & -0.681107 & 4.351379 & 3.471909 \\
$\mathrm{H}$ & 0.978165 & 3.733444 & 3.744003 \\
$\mathrm{H}$ & 1.096054 & 4.357681 & -0.807129 \\
$\mathrm{C}$ & 0.150970 & 3.564743 & -2.451111 \\
$\mathrm{O}$ & -0.616623 & 2.732620 & -2.935577 \\
$\mathrm{C}$ & 0.611313 & 4.794083 & -3.234314 \\
$\mathrm{H}$ & 1.499481 & 5.286407 & -2.808975 \\
$\mathrm{H}$ & 2.461121 & -5.297745 & -0.846863 \\
$\mathrm{H}$ & 0.827469 & 4.490250 & -4.268448 \\
$\mathrm{H}$ & -0.211929 & 5.526779 & -3.271652 \\
$\mathrm{O}$ & -0.113464 & -3.457962 & -0.039383 \\
$\mathrm{C}$ & -1.045547 & -4.002991 & -2.239548 \\
$\mathrm{H}$ & -2.037430 & -3.575394 & -2.029648 \\
$\mathrm{H}$ & -0.852325 & -3.929352 & -3.320984 \\
$\mathrm{H}$ & -1.053864 & -5.068878 & -1.963852 \\
$\mathrm{O}$ & 1.323517 & -3.867645 & -1.808502 \\
$\mathrm{C}$ & 0.733154 & -4.552689 & 0.324265 \\
& 0.234177 & -4.361893 & -0.610764 \\
$\mathrm{H}$ & 0.570367 & -4.457489 & 0.148849 \\
\hline
\end{tabular}

(E)-enamide with $\mathrm{R}=\mathrm{H}$.

$\mathrm{E}=-749.03696$ a.u.

$\begin{array}{lrrr}\mathrm{C} & 1.505178 & -0.564995 & -0.193431 \\ \mathrm{C} & 0.141546 & -0.581302 & -0.282026 \\ \mathrm{C} & -0.758894 & -0.048829 & -1.341505 \\ \mathrm{C} & -1.727943 & 1.179949 & -1.003742 \\ \mathrm{C} & -2.829604 & 0.446275 & -1.840445 \\ \mathrm{C} & -2.036371 & -0.877584 & -1.692387 \\ \mathrm{C} & -1.253385 & 2.538375 & -1.524437 \\ \mathrm{C} & -2.105125 & 1.268837 & 0.482303 \\ \mathrm{H} & -0.351616 & -1.065233 & 0.565985 \\ \mathrm{H} & -0.211665 & 0.198499 & -2.259846 \\ \mathrm{H} & -2.849540 & 0.799518 & -2.885033 \\ \mathrm{H} & -3.857519 & 0.473676 & -1.443186 \\ \mathrm{H} & -1.966722 & -1.539675 & -2.569894 \\ \mathrm{H} & -2.380973 & -1.476130 & -0.833595 \\ \mathrm{H} & -2.035129 & 3.307450 & -1.391287 \\ \mathrm{H} & -0.353569 & 2.878835 & -0.983626 \\ \mathrm{H} & -1.005771 & 2.493036 & -2.598341 \\ \mathrm{H} & -2.905748 & 2.015463 & 0.624787 \\ \mathrm{H} & -2.473066 & 0.309048 & 0.880513 \\ \mathrm{H} & -1.242325 & 1.575186 & 1.097320 \\ \mathrm{~N} & 2.224811 & -1.134234 & 0.885162 \\ \mathrm{C} & 2.495781 & 0.012738 & -1.158220 \\ \mathrm{O} & 3.713562 & -0.090981 & -0.991702 \\ \mathrm{O} & 1.954876 & 0.667462 & -2.215130 \\ \mathrm{C} & 2.923682 & 1.214163 & -3.141401 \\ \mathrm{H} & 2.328127 & 1.709334 & -3.917885 \\ \mathrm{H} & 3.540111 & 0.412722 & -3.574517 \\ \mathrm{H} & 3.578816 & 1.936071 & -2.632399 \\ \mathrm{H} & 3.235018 & -1.045508 & 0.755203 \\ \mathrm{C} & 1.754954 & -1.766583 & 2.019168 \\ \mathrm{O} & 0.558542 & -1.937130 & 2.278466 \\ \mathrm{C} & 2.853795 & -2.255416 & 2.962015 \\ \mathrm{H} & 3.876716 & -2.038559 & 2.617386 \\ \mathrm{H} & 2.705207 & -1.790771 & 3.949169 \\ \mathrm{H} & 2.746888 & -3.343122 & 3.096041 \\ & & & \end{array}$




$\begin{array}{cccc}\text { (E)-8 } & & & \\ \mathrm{E}=-1055.46811 & \mathrm{a} & & \\ \mathrm{C} & -2.572973 & 0.041922 & -0.179235 \\ \mathrm{C} & -1.235648 & 0.310020 & -0.091085 \\ \mathrm{C} & -0.064876 & -0.605771 & -0.167098 \\ \mathrm{C} & 0.972914 & -0.648560 & 1.048425 \\ \mathrm{C} & 2.100659 & -0.776242 & -0.052728 \\ \mathrm{C} & 1.121461 & -0.183570 & -1.092501 \\ \mathrm{C} & 0.796856 & -1.851134 & 1.982349 \\ \mathrm{C} & 0.999683 & 0.656454 & 1.858485 \\ \mathrm{H} & -1.004816 & 1.369698 & 0.049788 \\ \mathrm{H} & -0.362613 & -1.635411 & -0.398205 \\ \mathrm{H} & 2.250183 & -1.850342 & -0.262435 \\ \mathrm{C} & 3.512678 & -0.183850 & 0.077297 \\ \mathrm{H} & 1.130975 & -0.602485 & -2.110468 \\ \mathrm{H} & 1.216735 & 0.911595 & -1.150619 \\ \mathrm{H} & 1.614327 & -1.908031 & 2.722523 \\ \mathrm{H} & -0.151300 & -1.775164 & 2.542671 \\ \mathrm{H} & 0.782300 & -2.801522 & 1.422183 \\ \mathrm{H} & 1.819930 & 0.643509 & 2.595012 \\ \mathrm{H} & 1.144834 & 1.546100 & 1.229656 \\ \mathrm{H} & 0.053269 & 0.776782 & 2.412365 \\ \mathrm{~N} & -3.574591 & 1.040244 & -0.095613 \\ \mathrm{C} & -3.254121 & -1.278364 & -0.381167 \\ \mathrm{O} & -4.480954 & -1.378470 & -0.462904 \\ \mathrm{H} & -2.427460 & -2.349546 & -0.465594 \\ \mathrm{H} & -3.101499 & -3.613745 & -0.669802 \\ \mathrm{H} & 3.206724 & 2.803709 & -0.919015 \\ \mathrm{H} & -2.299259 & -4.360407 & -0.713523 \\ \mathrm{H} & -3.672731 & -3.599862 & -1.609654 \\ \mathrm{H} & -3.786568 & -3.825431 & 0.164132 \\ \mathrm{H} & -4.512177 & 0.644891 & -0.194853 \\ \mathrm{C} & -3.446598 & 2.401772 & 0.093336 \\ \mathrm{O} & -2.367945 & 2.991232 & 0.222629 \\ \mathrm{C} & -4.776875 & 3.153311 & 0.135683 \\ \mathrm{H} & -5.662856 & 2.516238 & -0.010752 \\ \mathrm{H} & -4.863970 & 3.664233 & 1.107343 \\ \mathrm{H} & -4.767082 & 3.933214 & -0.641388 \\ \mathrm{O} & 3.444245 & 1.246989 & 0.187349 \\ \mathrm{C} & 4.321703 & -0.787862 & 1.232888 \\ \mathrm{C} & 4.238655 & -0.464300 & -1.154804 \\ \mathrm{H} & 4.525401 & 0.706867 & -2.284394\end{array}$



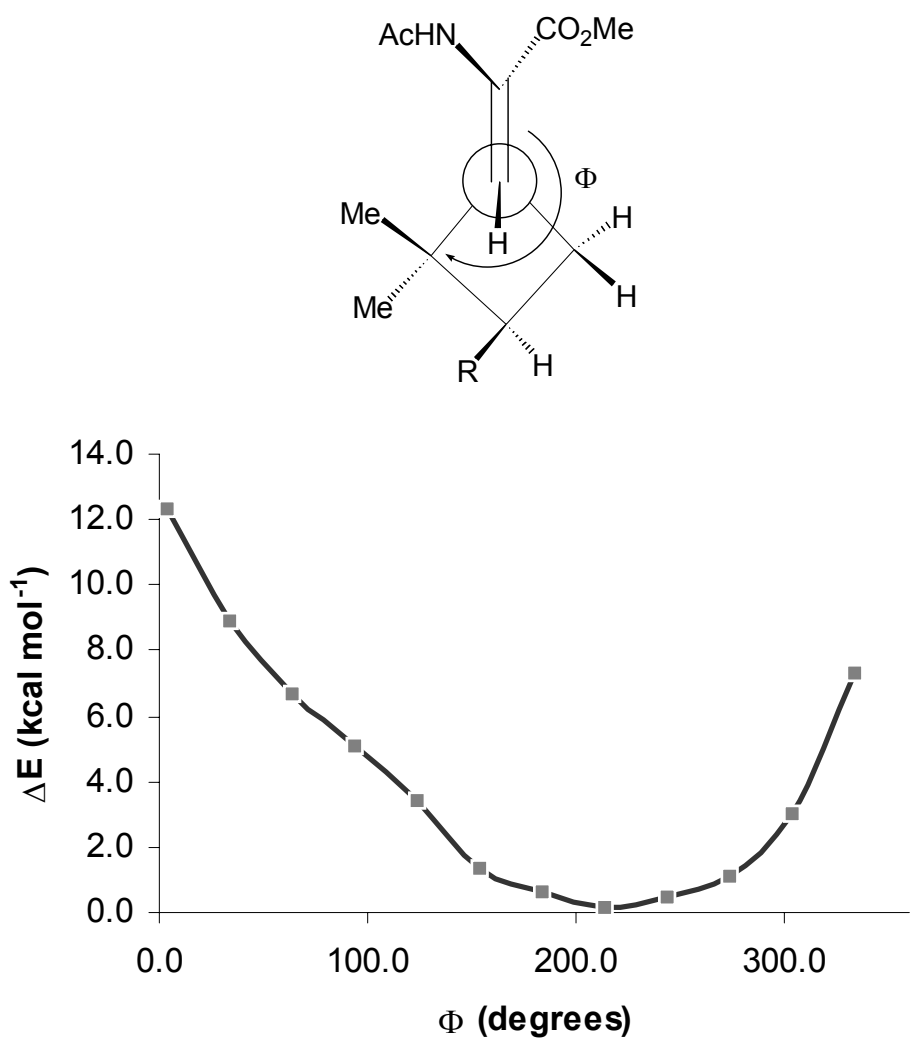

Figure S1. Energy curve corresponding to rotation around $\mathrm{C}_{3}-\mathrm{C}_{4}$ bond obtained at the AM1 level of calculation for a model $(E)$-enamide with $\mathrm{R}=\mathrm{H}$. 